\title{
Prime Fuzzy Bi-Ideals in Near-Subtraction Semigroups
}

\author{
K. Mumtha \\ Research Scholarof Mathematics \\ A.P.C.Mahalaxmi College for Women \\ Thoothukudi, India. \\ mamthakasi8696@gmail.com
}

\author{
V. Mahalakshmi \\ Assistant Professor of Mathematics \\ A.P.C.Mahalaxmi College for Women \\ Thoothukudi, India. \\ mahalakshmi@apcmcollege.ac.in
}

\author{
S. Usha Devi \\ Assistant Professor of Mathematics \\ Sri Parasakthi College for Women \\ Courtallam, India. \\ ushadevinathan@gmail.com
}

Abstract - A study on fuzzy prime ideals in near-subtraction semigroups is already known. We have to expand the concept of prime fuzzy bi-ideals in near-subtraction semigroups and analyse some of its properties to characterize it. This will lead to learn a new type of fuzzy ideal and to develope the researcher to made their research.

Keywords - Fuzzy Ideals, Fuzzy prime ideals.

\section{INTRODUCTION}

In 1965, fuzzy set was first introduced by L.A.Zadeh [7]. The notion of Near-subtraction semigroup was studied by B.M.Schein. K.H.Kim et [2] \& they established the concept of Ideals in near-subtraction semigroup \& fuzzy set. Prince Williams [3] described the concept of Fuzzy ideals. Similarly, the concept such as Fuzzy bi-ideals has been described by V.Chinnadurai et. al. A detailed study on Fuzzy prime ideals was carried out by Mumtha.K and Mahalakshmi.V [6]. In this paper, we explore the concept of prime fuzzy bi-ideals in nearsubtraction semigroups and discuss some of its properties.

\section{PRELILIMINARIES}

Definition: 2.1

A right near-subtraction semigroup is a non-empty set X with “-" \& "." satisfies:

(i) $(X,-)$ is a subtraction algebra

(ii) $(X, \cdot)$ is a semigroup

(iii) For all $p, q, r \in X,(p-q) \cdot r=p \cdot r-q \cdot r$ (right distributive law)

Definition: 2.2

If $p .0=0 . p=0$, for all $p \in X$, then $\mathrm{X}$ is a zerosymmetric and is denoted by $X_{0}$. Now after, $\mathrm{X}$ stands for a zero-symmetric right near-subtraction semigroup $(X,-, \cdot)$ with at least two elements.

Definition: 2.3

A fuzzy subset is the mapping $\mu$ from the nonempty set $\mathrm{X}$ into the unit interval $[0,1]$.

Definition: 2.4

A fuzzy subset $\mu$ of $\mathrm{X}$ is called a fuzzy ideal of $\mathrm{X}$ if

(i) $\mu(x-y)=\min \{\mu(x), \mu(y)\}$.

(ii) $\mu(x y) \geq \mu(y)$,

(iii) $\mu(x y) \geq \mu(x)$, for every $x, y \in X$.

Definition: 2.5

A fuzzy ideal $\mu$ is called a fuzzy prime ideal of $\mathrm{X}$ if $\sigma . \delta \subseteq \mu \Rightarrow \sigma \subseteq \mu$ or $\delta \subseteq \mu$, where $\sigma \& \delta$ are any two fuzzy ideals of $\mathrm{X}$.
Definition: 2.6

Let $\mu$ and $\lambda$ be any two fuzzy subsets of $X$. Then $\mu \cap \lambda, \mu \cup \lambda, \mu \lambda, \lambda \mu, \mu * \lambda$ are fuzzy subsets of $\mathrm{X}$ that are defined by,

$$
\begin{aligned}
& (\mu \cap \lambda)(x)=\min \{\mu(x), \lambda(x)\} \\
& (\mu \cup \lambda)(x)=\max \{\mu(x), \lambda(x)\}
\end{aligned}
$$

$$
\begin{aligned}
(\mu-\lambda)(x) & =\left\{\begin{array}{lr}
\sup _{x=y-z} \min \{\mu(y), \lambda(z)\} & \text { if } x=y-z \\
0 & \text { otherwise }
\end{array}\right. \\
\mu \lambda(x) & = \begin{cases}\sup _{x=y z} \min \{\mu(y), \lambda(z)\} & \text { if } x=y z \\
0 & \text { otherwise }\end{cases}
\end{aligned}
$$

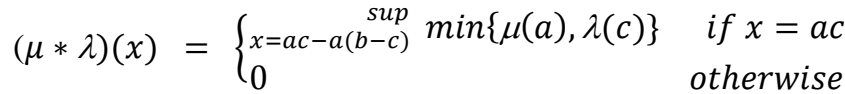

Definition: 2.7

For any fuzzy set $\mu$ in $X$ and $t \in[0,1]$. We define $U(\mu ; t)=\{x / x \in X, \mu(x) \geq t\}$, which is called a upper $t$ level cut of $\mu$.

Definition: 2.8

Let $I \subseteq X$. Define a function $f_{I}: \mathrm{X} \rightarrow[0,1]$ by,

$f_{I}(x)=\left\{\begin{array}{cc}1 & \text { if } x \in I \\ 0 & \text { otherwise }\end{array}\right.$, for every $x \in X$.

Clearly, $f_{I}$ is a fuzzy subset of $\mathrm{X}$ and it is called the characteristic function of I.

Definition: 2.9

A fuzzy ideal $\mu$ of $\mathrm{X}$ is said to be normal if there exists $a \in X$ such that $\mu(a)=1$

Definition: 2.10

A fuzzy ideal $\mu$ of $\mathrm{X}$ is said to be weakly complete if it is normal and there exists $z \in X$ such that $\mu(z)<1$.

Theorem: 2.11

Let $\mu$ be a fuzzy bi-ideal of $X$. Then the finitely generated set, $X_{\mu}=\{x \in X / \mu(x)=\mu(0)\}$ is an bi-ideal of $\mathrm{X}$.

\section{Theorem: 2.12}

Let $\mathrm{A}$ be a non-empty subset and $\mu_{A}$ be a fuzzy set in $\mathrm{X}$ defined by, $\mu_{A}(x)=\left\{\begin{array}{cc}1 & \text { if } x \in A \\ S & \text { otherwise }\end{array}, \forall x \in X\right.$ and $s \in[0,1)$. Then $\mu_{A}$ is a fuzzy bi-ideal of $\mathrm{X}$ iff $\mathrm{A}$ is an bi-ideal of X. Moreover, $X_{\mu_{A}}=A$. 
Lemma: 2.13

Let $\chi_{A}$ be the characteristic function of a subset

$A \subset X$. Then $\chi_{A}$ is a fuzzy bi-ideal of $\mathrm{X}$ iff $\mathrm{A}$ is an bi-ideal of $\mathrm{X}$.

\section{PRIME FUZZY BI-IDEALS}

\section{Definition: 3.1}

A fuzzy bi-ideal $f$ is called a prime fuzzy bi-ideal of $X$ if for any two fuzzy bi-ideals $g \& h$ of $X$ such that $g . h \leq f \Rightarrow g \leq f$ (or) $h \leq f$.

E.g: 3.1.1

Let $X=\{0,1,2,3\}$ with " - " $\&$ ". " are defined as,

\begin{tabular}{l|lllll|llll}
- & $\mathbf{0}$ & $\mathbf{1}$ & $\mathbf{2}$ & $\mathbf{3}$ & & $\mathbf{0}$ & $\mathbf{1}$ & $\mathbf{2}$ & $\mathbf{3}$ \\
\hline $\mathbf{0}$ & 0 & 0 & 0 & 0 & $\mathbf{0}$ & 0 & 0 & 0 & 0 \\
$\mathbf{1}$ & 1 & 0 & 1 & 0 & $\mathbf{1}$ & 0 & 1 & 0 & 1 \\
$\mathbf{2}$ & 2 & 2 & 0 & 0 & $\mathbf{2}$ & 0 & 0 & 2 & 2 \\
$\mathbf{3}$ & 3 & 2 & 1 & 0 & $\mathbf{3}$ & 0 & 1 & 2 & 3
\end{tabular}

Let $f, g \& h$ be fuzzy subsets of $\mathrm{X}$ such that,

$$
\begin{aligned}
& f(0)=1, \quad f(1)=0.8, \quad f(2)=0.7, \quad f(3)=0.5 \\
& g(0)=1, \quad g(1)=0.8, \quad g(2)=0.6, \quad g(3)=0.3 \\
& h(0)=1, \quad h(1)=0.7, \quad h(2)=0.5, \quad h(3)=0.2
\end{aligned}
$$

Clearly, $f$ is prime fuzzy bi-ideal of $\mathrm{X}$.

E.g: 3.1.2

Let $X=\{0,1,2,3\}$ with " - "\&". " are defined as ,

\begin{tabular}{l|llll}
- & $\mathbf{0}$ & $\mathbf{1}$ & $\mathbf{2}$ & $\mathbf{3}$ \\
\hline $\mathbf{0}$ & 0 & 0 & 0 & 0 \\
$\mathbf{1}$ & 1 & 0 & 3 & 2 \\
$\mathbf{2}$ & 2 & 0 & 0 & 2 \\
$\mathbf{3}$ & 3 & 0 & 3 & 0
\end{tabular}

\begin{tabular}{l|llll} 
& $\mathbf{0}$ & $\mathbf{1}$ & $\mathbf{2}$ & $\mathbf{3}$ \\
\hline $\mathbf{0}$ & 0 & 0 & 0 & 0 \\
$\mathbf{1}$ & 0 & 1 & 2 & 3 \\
$\mathbf{2}$ & 0 & 0 & 0 & 0 \\
$\mathbf{3}$ & 0 & 1 & 2 & 3
\end{tabular}

Let $f, g \& h$ be fuzzy subsets of X such that,

$$
\begin{array}{llll}
f(0)=1, & f(1)=0.4, & f(2)=0.4, & f(3)=1 \\
g(0)=0.8, & g(1)=0, & g(2)=0.8, & g(3)=0 \\
h(0)=0.8, & h(1)=0, & h(2)=0.8, & h(3)=0
\end{array}
$$

Here $g . h \leq f$ but neither $g \leq f$ nor $h \leq f$, for some $x \in X$. Clearly, $f$ is not a prime fuzzy bi-ideal of $\mathrm{X}$.

\section{Theorem: 3.2}

Intersection of all prime fuzzy bi-ideals of $\mathrm{X}$ is also a prime fuzzy bi-ideal of $X$.

\section{Proof:}

Let $\left\{f_{i} / i \in \Omega\right\}$ be the set of all prime fuzzy bi-ideals in $\mathrm{X}$.

To prove: $f=\bigcap_{i \in \Omega} f_{i}$ is also a prime fuzzy bi-ideal.

Let $g \& h$ be any fuzzy bi-ideals of X such that g. $h \leq \bigcap_{i \in \Omega} f_{i} \Rightarrow g . h \leq f_{i}$, for all $i \in \Omega$.

Since each $f_{i}$ is a prime fuzzy b-ideal.

Therefore, $g \leq f_{i}$ (or) $h \leq f_{i}$, for all $i \in \Omega$.

(i.e) $g \leq \bigcap_{i \in \Omega} f_{i}$ (or) $h \leq \bigcap_{i \in \Omega} f_{i}$.

Note: 3.3

Every fuzzy prime ideal is a prime fuzzy bi-ideal but the converse need not be true in general.

\section{Theorem: 3.4}

If $f$ is a prime fuzzy bi-ideal of $\mathrm{X}$ then the finitely generated set is a prime bi-ideal of $X$.

Proof:

Assume that $f$ is a prime fuzzy bi-ideal of $X$.

By Theorem 2.11, $X_{f}$ is a bi-ideal of $\mathrm{X}$.

To prove: $X_{f}$ is a prime bi-ideal of $\mathrm{X}$.

Let $\mathrm{A} \& \mathrm{~B}$ be any two bi-ideals in $\mathrm{X}$ such that $A B \subseteq X_{f}$.

We have to prove $A \subseteq X_{f}$ or $B \subseteq X_{f}$.

Define the fuzzy subsets $g \& h$ of $\mathrm{X}$ as,

$$
g(x)=\left\{\begin{array}{ll}
f(0) & \text { if } x \in A \\
0 & \text { if } x \notin A
\end{array} \quad h(y)= \begin{cases}f(0) & \text { if } y \in B \\
0 & \text { if } y \notin B\end{cases}\right.
$$

By Theorem 2.12, $g \& h$ are fuzzy bi-ideals.

Next we verify that $g . h \leq f$.

Since $g \cdot h(a)= \begin{cases}\sup _{a=b c}^{\sin }\{\min \{(b), h(c)\}\} & \text { if } a=b c \\ 0 & \text { otherwise }\end{cases}$

$\Rightarrow g(b)=h(c)=f(0)$. So $b \in A \& c \in B$.

Now, $a=b c \in A B \subseteq X_{f}$. (i.e) $\in X_{f} \Rightarrow f(a)=f(0)$.

Hence, $g . h(a) \leq f(a), \forall a \in X$. Thus $g . h \leq f$.

Since $f$ is a prime fuzzy bi-ideal,

So we have that $g \leq f$ or $h \leq f$.

Suppose $g \leq f$. If $A \not \subset X_{f}$, then there exists $a \in A$ such that $a \notin X_{f}$. This means that $f(a) \neq f(0)$. Already We know that, $f(0) \geq f(a)$. But $f(0) \neq f(a)$ andso $f(0)>f(a)$.

Now, $g(a)=f(0)>f(a)$.

Which is a contradiction to $g \leq f$. Hence $A \subseteq X_{f}$.

Similarly, If $h \leq f$, then we can show that $B \subseteq X_{f}$.

This shows that $X_{f}$ is a prime bi-ideal of $\mathrm{X}$.

\section{Theorem: 3.5}

Let $\mathrm{I}$ be an bi-ideal of $\mathrm{X}$ and $f$ be a fuzzy set in $\mathrm{X}$ defined by, $f(x)=\left\{\begin{array}{cc}1 & \text { if } x \in I \\ S & \text { otherwise }\end{array}, \forall x \in X \& s \in[0,1)\right.$. If $\mathrm{I}$ is a prime bi-ideal of $\mathrm{X}$ then $f$ is a prime fuzzy bi-ideal of $\mathrm{X}$.

\section{Proof:}

Suppose $\mathrm{I}$ is a prime ideal of $\mathrm{X}$.

To prove: $f$ is a prime fuzzy bi-ideal of $\mathrm{X}$. 
By Theorem 2.12, $f$ is a fuzzy bi-ideal of X. Let $g \& h$ be two fuzzy ideals of $\mathrm{X}$ such that $g . h \leq f$.

To prove: $g \leq f$ or $h \leq f$.

Suppose not, (i.e) $g \not f$ and $h \not f$.

Then $g(x)>f(x)$ and $h(y)>f(y), \forall x, y \in X$.

Now, $f(x) \neq 1$ and $f(y) \neq 1$

$\Longrightarrow f(x)=f(y)=s$ andso $x, y \notin I$.

Since $\mathrm{I}$ is a prime ideal, we have that $\langle x\rangle\langle y\rangle \not \subset I$.

Then $f(a)=s$ and hence $g \cdot h(a) \leq f(a)=s$.

Since $a=c d$, where $c=\langle x\rangle \& d=\langle y\rangle$.

Then, $s=f(a) \geq g \cdot h(a)$.

$$
\text { Now, } \begin{aligned}
g \cdot h(a)=\sup _{a=c d} & \{\min \{g(c), h(d)\}\} \\
& \geq \min \{g(c), h(d)\} \\
& \geq \min \{g(x), h(y)\} \\
& >\min \{f(x), f(y)\}=s
\end{aligned}
$$

Therefore $g \cdot h(a)>s$. Which is a contradiction.

Hence, $f$ is a prime fuzzy bi-ideal of $\mathrm{X}$.

\section{Corollary : 3.6}

Let $\chi_{P}$ be the characteristic function of a subset

$P \subseteq X$. Then $\chi_{P}$ is a prime fuzzy bi-ideal iff $\mathrm{P}$ is a prime bi-ideal of $X$.

\section{Theorem: 3.7}

If $f$ is a prime fuzzy bi-ideal of $\mathrm{X}$ then, $f(0)=1$.

Proof:

Suppose $f$ is a prime fuzzy bi-ideal of $\mathrm{X}$.

To prove: $f(0)=1$.

Suppose not, (i.e) $f(0)<1$.

Since $f$ is not a constant, then there exists $a \in X$ such that $f(a)<f(0)$

Define the fuzzy subsets $g \& h$ as, $\forall x \in X$

$g(x)=f(0)$ and $h(x)=\left\{\begin{array}{lr}1 & \text { if } f(x)=f(0) \\ 0 & \text { otherwise }\end{array}\right.$

Since $g$ is a constant function, $g$ is a fuzzy bi-ideal.

Note that, $h$ is the characteristics function of $X_{f}$.

Now, by Theorem: $2.12, h$ is the fuzzy bi-ideal of $X$.

Since $h(0)=1>f(0)$ and $g(a)=f(0)>f(a)$

We have that, $g \$ f \& h \nsubseteq f$.

Let $b \in X$. We know that,

$$
g \cdot h(b)=\left\{\begin{array}{lr}
\sup _{b=c d}\{\min \{\sigma(c), \delta(d)\}\} & \text { if } b=c d \\
0 & \text { otherwise }
\end{array}\right.
$$

Now, we prove, $\min \{g(c), h(d)\} \leq f(b)$, where $b=c d$.

For this, we consider two cases, $h(x)=0 \& h(x)=1$ in the following:
Case - (i)

Suppose $h(x)=0$.

Then $h(x)<h(0)$ (By definition of $h$ ). Now, $\min \{g(c), h(d)\}=\min \{f(0), 0\}=0 \leq f(x y)=f(b)$.

Case - (ii)

Suppose $h(x)=1$. Then $f(x)=f(0)$.

Now, $\min \{g(c), h(d)\}=\min \{f(0), 1\}=f(0)=f(x)$

$$
\leq f(x y)=f(b) \text {. }
$$

From this, we conclude that,

$g \cdot h(b)=\min \{g(c), h(d)\} \leq f(b)$ andso $g . h \leq f$.

Since, $f$ is a prime fuzzy bi-ideal, we have $g \leq f$ or $h \leq f$.

Which is a contradiction to $g \$ f$.

Hence, $f(0)=1$.

\section{Theorem: 3.8}

Every prime fuzzy bi-ideal is normal.

\section{Proof:}

By Previous Theorem 3.7, it is obviously true.

\section{Theorem: 3.9} normal

Every prime fuzzy bi-ideal is weakly completely

\section{Proof:}

Let $f$ be prime fuzzy bi-ideal.

Then $f$ is normal and $f$ lies between the values $0 \& 1$. It follows that, $f(0)=1 \& f(x)<1$, for all $x \in X$. Therefore, $f$ is weakly completely normal.

\section{Theorem: 3.10}

If $f$ is a prime fuzzy bi-ideal of $\mathrm{X}$ then,

$|\operatorname{Im}(f)|=2$. Moreover, $\operatorname{Im}(f)=\{1, s\}$, where $0 \leq s<1$.

\section{Proof:}

Suppose $f$ is a prime fuzzy bi-ideal of X.

To prove: $\operatorname{Im}(f)$ contains exactly two values.

We know that, by previous Theorem 3.7, $f(0)=1$.

Let $a \& b$ be two elements of $\mathrm{X}$ such that,

$$
f(a)<1 \text { and } f(b)<1 .
$$

Enough to prove: $f(a)=f(b)$.

Part-(i)

Define the fuzzy subsets $g$ and $h$ as, $\forall x \in X$ and $a \in X$ $g(x)=f(a)$ and $h(x)=\left\{\begin{array}{lr}1 & \text { if } x \in<a> \\ 0 & \text { otherwise }\end{array}\right.$ By Theorem: 2.12, $g \& h$ are fuzzy bi-ideals of $X$. Since $a \in\langle a\rangle$, we have $h(a)=1\rangle f(a)$ andso $g \not f$. Let $z \in X$. We know that,

$$
g \cdot h(z)= \begin{cases}\sup _{z=x y}^{\sin }\{\min \{g(x), h(y)\}\} & \text { if } z=x y \\ 0 & \text { otherwise }\end{cases}
$$


If $x \notin\langle a>$, then $h(x)=0$

$\Longrightarrow \min \{g(x), h(y)\}=\min \{f(a), 0\}=0 \leq f(x y)=f(z)$.

If $x \in\langle a\rangle$, then $h(x)=1$

$\Rightarrow \min \{g(x), h(y)\}=\min \{f(a), 1\}=f(a) \leq f(x y)$

$$
=f(z) \text {. }
$$

We know that, $f(x) \geq f(a)$, for all $x \in\langle a\rangle$

It follows that, $f(a) \leq f(x) \leq f(x y)=f(z)$.

From these, we conclude that, $g . h \leq f$.

Since $f$ is a prime fuzzy bi-ideal, we have $g \leq f$ or $h \leq f$

Since $h \not f$. It follows that $g \leq f$.

Now, $f(b) \geq g(b)=f(a)$.

Part-(ii)

Now, we construct fuzzy bi-ideals $\rho \& \theta$ of X,

$\rho(x)=f(b)$ and $\theta(x)=\left\{\begin{array}{lr}1 & \text { if } x \in<b> \\ 0 & \text { otherwise }\end{array}, \forall x \in X\right.$

As in part-(i), we can verify that $f(a) \geq f(b)$.

Thus from parts-(i) \& (ii), it follows that $f(a)=f(b)$.

Hence the proof.

\section{Theorem: 3.11}

Let $f$ be fuzzy bi-ideal in $\mathrm{X}$. Then $f$ is a prime fuzzy bi-ideal of $\mathrm{X}$ iff each level subset $f_{t}, t \in \operatorname{Im}(f)$ of $f$ is a prime bi-ideal of $\mathrm{X}$.

Proof:

Assume that $f$ is a prime fuzzy bi-ideal of $\mathrm{X}$.

By Theorem 3.7, $f_{t}$ is an bi-ideal of $\mathrm{X}$.

To prove: $f_{t}$ is a prime bi-ideal of $\mathrm{X}$.

Let $\mathrm{A} \& \mathrm{~B}$ be two ideals in $\mathrm{X}$ such that $A B \subseteq f_{t}$.

Define the fuzzy subsets $g \& h$ of $\mathrm{X}$ as,

$$
g(x)=\left\{\begin{array}{ll}
1 & \text { if } x \in A \\
0 & \text { otherwise }
\end{array} \text { and } h(x)= \begin{cases}1 & \text { if } x \in B \\
0 & \text { otherwise }\end{cases}\right.
$$

By Theorem 2.12, $g \& h$ are fuzzy bi-ideals of X.

Next we verify that, $g \cdot h \leq f$.

Since, $g \cdot h(a)=\left\{\begin{array}{lc}\sup _{a=b c}\{\min \{g(b), h(c)\}\} & \text { if } a=b c \\ 0 & \text { otherwise }\end{array}\right.$

We conclude that, $g(b)=h(c) \geq t$. So $b \in A \& c \in B$.

Now, $a=b c \in A B \subseteq f_{t}$. (i.e) $a \in f_{t} \Rightarrow f(a) \geq t$.

Hence $g . h(a) \leq f(a), \forall a \in X$. Thus $g . h \leq f$.

Since $f$ is prime fuzzy bi-ideal, we have $g \leq f$ or $h \leq f$.

Suppose $g \leq f$. If $A \nsubseteq f_{t}$, then there exists $a \in A$ such that $a \notin f_{t}$. This means that $f(a) \geq t$. (i.e) $f(a)<t$.

Now, $g(a) \geq t>f(a)$. Which is a contradiction to $g \leq f$.

Similarly, If $h \leq f$, then we can show that $B \subseteq f_{t}$.

This shows that $f_{t}$ is a prime bi-ideal of $\mathrm{X}$.

\section{Conversely,}

Assume that $f_{t}, t \in \operatorname{Im}(f)$ is a prime bi-ideal of X. To prove: $f$ is a prime fuzzy bi-ideal of $\mathrm{X}$.

Let $f$ be a fuzzy set in $\mathrm{X}$ defined by,

$$
f(x)=\left\{\begin{array}{cc}
1 & \text { if } x \in f_{t} \\
s & \text { otherwise }
\end{array}\right.
$$

By Theorem 2.12, $f$ is an fuzzy bi-ideal of $\mathrm{X}$.

To prove: $f$ is prime.

Let $g \& h$ be two fuzzy bi-ideals of X such that $g . h \leq f$.

Enough To prove: $g \leq f$ or $h \leq f$.

Suppose $g \not f$ and $h \not f$.

Then $g(x)>f(x)$ and $h(y)>f(\mathrm{y}), \forall x \in X$.

Now, $f(x) \neq 1$ and $f(y) \neq 1$

$\Longrightarrow f(x)=f(y)=s$ and also $x, y \notin f_{t}$.

Since $f_{t}$ is a prime ideal, we have that $\langle x\rangle\langle y\rangle \not \subset f_{t}$.

Then $f(a)=s$ and hence $g \cdot h(a) \leq f(a)=s$.

Since $a=c d, c=\langle x\rangle \& d=\langle y\rangle$. Then $s=f(a) \geq g \cdot h(a)$.

Now, $g \cdot h(a)=\min \{g(c), h(d)\}$

$$
\begin{aligned}
& \geq \min \{g(c), h(d)\} \\
& \geq \min \{g(x), h(y)\} \\
> & \min \{f(x), f(y)\}=s .
\end{aligned}
$$

Therefore, $g \cdot h(a)>s$. Which is a contradiction.

Hence $f$ is a prime fuzzy bi-ideal of $\mathrm{X}$.

\section{Theorem: 3.12}

Let $\mathrm{P}$ be a prime bi-ideal of $\mathrm{X}$ and $\alpha$ be a prime element of $L, L \in[0,1]$. Let $f$ be a fuzzy subset of $\mathrm{X}$ defined by, $f(x)=\left\{\begin{array}{cc}1 & \text { if } x \in I \\ 0 & \text { otherwise }\end{array}\right.$ iff $f$ is a prime fuzzy bi-ideal of $\mathrm{X}$.

Proof:

Clearly, $f$ is a non-constant fuzzy bi-ideal.

To prove: $f$ is prime.

Let $g \& h$ be two fuzzy bi-ideals such that, $g \nsubseteq f$ and $h \nsubseteq f$. Then there exists $x, y \in X$ such that $g(x) \$ f(x)$ and $h(y) \$ f(y)$.

This implies that $f(x)=f(y)=\alpha$ and hence $x, y \notin I$. Since $\mathrm{I}$ is prime, then there exists an element $\mathrm{r}$ in $\mathrm{X}$ such that $x r y \notin I$.

Now, we have $f(x) \$ \alpha \& f(r y) \$ \alpha$ (otherwise $h(y) \$ \alpha)$ and since $\alpha$ is prime, $g(x) . h(x y) \$ \alpha$ and hence $g . h(x r y) \not \alpha=f(x r y)$ so that $g . h \$ f$.

Hence $f$ is prime fuzzy bi-ideal.

Conversely,

Let $f$ be a prime fuzzy bi-ideal. Then, $f(0)=1$.

Next we observe that $f$ assumes exactly two values.

Let $a \& b$ be elements of X such that $f(a)<1 \& f(b)<1$. 
Define $g$ \& $h$ as, $g(x)=\left\{\begin{array}{cc}1 & \text { if } x \in\langle a\rangle \\ o & \text { otherwise }\end{array}\right.$ and

$$
h(x)=f(a), \forall x \in X .
$$

By Theorem: 2.12, $g \& h$ are fuzzy bi-ideals.

And also we have, $g(x) \cdot h(y) \leq f(x y), \forall x, y \in X$.

And hence $g . h \leq f$. Put $g \$ f$. Since $g(a)=1>f(a)$. Since $f$ is prime fuzzy bi-ideal andso $h \leq f$ so that

$h(b) \leq f(b)$ hence $f(a) \leq f(b)$. Thus $f$ assumes only one value, say $\alpha$ other than 1 .

Let $I=\{x \in X / f(x)=1\}$. Then clearly, I is a proper bi-ideal of $X$ and for $x \in X, f(x)=\left\{\begin{array}{cc}1 & \text { if } x \in I \\ \alpha & \text { otherwise }\end{array}\right.$.

Now, to prove: I is a prime bi-ideal of $\mathrm{X} \& \alpha$ is a prime element in L.

That $\alpha$ is prime follows that the fact that for any $a \in L \&$ for the constant map $\bar{a} \leq f$ iff $a \leq \alpha$. Let $J \& K$ be ideals of $\mathrm{X}$ such that $J K \subseteq I$. Then $\chi_{J} \chi_{K}=\chi_{J K} \subseteq \chi_{I} \subseteq f$ so that $\chi_{J} \subseteq f$ or $\chi_{K} \subseteq f$. Which implies that $J \subseteq I$ or $K \subseteq I$.

\section{Corollary: 3.13}

Let $\mathrm{L}$ be a complete chain and $\mathrm{P}$ is an bi-ideal of $\mathrm{X}$. Then $\mathrm{P}$ is a prime bi-ideal of $\mathrm{X}$ iff $\chi_{P}$ is a prime fuzzy bi-ideal of X..

\section{CONCLUSION}

We have analyse the concept of prime fuzzy bi-ideal $f$ in near-subtraction semigroups and investigated some of its properties. We find

- $f(0)=1$

- $\operatorname{Im}(f)=\{1, s\}$, where $0 \leq s<1$.

- Prime fuzzy bi-ideal iff each level subset is prime fuzzy bi-ideal.

\section{ACKNOWLEDGEMENT}

We thank the anonymous referees for their useful suggestions.

\section{REFERENCES}

[1] B.Zelinka, "Subtraction semigroups", Math. Bohemica. 120 (1995), pp. 445-447.

[2] B. M. Schein, "Difference semigroups", Communications in algebra 20 (1992), pp. 21532169.

[3] D.R.Prince Williams, "fuzzy ideals in nearsubtraction semigroups", International journal of computational \& mathematical sciences, 2 (2008) pp. 39-46.

[4] G.Pilz, "Near-ring, The theory and its applications". Second edition. North-Holland Mathematics Studies, 23. North-Holland Publishing Co., Amsterdam, 1983. xv+470 pp. ISBN:0 7204-0566-1.

[5] J.B.Jun and K.H.Kim, "On ideals in subtraction algebras", Sc. Math. Jpn. 65 (2007), pp. 129134.

[6] K.Mumtha and V.Mahalakshmi, "Fuzzy prime ideals in near-subtraction semigroups", 5 (2020) pp. 269-277.

[7] L.A.Zadeh, "Fuzzy sets", Information control 8 (1965), pp. 338-353.

[8] P.Dheena and G.Mohanraj, "Fuzzy weakly prime ideals of near-subtraction semigroup”, 4 (2012), pp. 235-242.

[9] P.Dheena and G.Satheeshkumar, "Weakly prime left ideals of near-subtraction semigroup", 23 (2008), pp. 325-331.

[10]P.Dheena and G.Satheeshkumar, "On prime \& fuzzy prime ideals of near-subtraction semigroup", 4 (2009), pp. 2345-2353.

\section{Creative Commons Attribution License 4.0 (Attribution 4.0 International, CC BY 4.0)}

This article is published under the terms of the Creative Commons Attribution License 4.0

https://creativecommons.org/licenses/by/4.0/deed.en US 